\title{
ANALYSIS OF EPIGENETIC MOLECULES SIRT2 E SIRT6 IN BREAST CANCER SUBTYPES
}

Joana D. R. Santos'1, Ananda C. F. Aguiar ${ }^{1}$, Glauber M. Leitão², Maria A. C. S. M. Borba', Nancy C. F. L. Ferreira², Darley L. F. Filho ${ }^{3}$, José L. L. Filho', ${ }^{1,4}$ Danyelly B. G. Martins ${ }^{1,4}$

'Molecular Prospection and Bioinformatics Group, Laboratory Keizo Asami of Immunopathology (LIKA), Universidade Federal de Pernambuco - Recife (PE), Brazil.

${ }^{2}$ Hospital das Clínicas - Professor Romero Marques, Universidade Federal de Pernambuco - Recife (PE), Brazil.

${ }^{3}$ Barão de Lucena Hospital - Recife (PE), Brazil.

${ }^{4}$ Department of Biochemistry, Universidade Federal de Pernambuco - Recife (PE), Brazil.

The aim of this study was to evaluate the gene expression of sirtuin 2 (SIRT2) and 6 (SIRT6) in 18 breast cancer fresh tissue samples of which 2 HER2+, 11 luminal B, and 5 luminal A. Clinical and staging data were considered. RNA was purified using the RNeasy Mini Kit (Qiagen), cDNA was prepared using QuantiTect Reverse Transcription (Qiagen). The analysis was performed by qPCR (quantitative polymerase chain reaction) in the real-time PCR systems StepOnePlus (Applied Biosystems) using the GoTaq ${ }^{\circledR}$ qPCR Master Mix kit (Promega). ß-actin was used as the reference gene to normalize the expression of SIRT2 and SIRT6 with specific primers. Correlations between tumor groups and clinicopathological features were analyzed using Mann-Whitney for independent samples and paired t test in GraphPad Prism 7.0 software. In luminal A and B groups, SIRT2 gene expression levels exhibited a correlation with the clinical-pathological staging scores, respectively, $\mathrm{p}=0.0265$ and $\mathrm{p}=0.0001$. HER2+ samples showed no correlation between SIRT2 and SIRT6 gene expression and staging. Characteristics such as age, obesity, smoking and alcoholism did not correlate with the genetic expression of the SIRTs in any of the breast cancer subtypes of this study. Sirtuins (SIRTs), class III histone deacetylases, are differentially expressed in several human cancers, where they display both oncogenic and tumor-suppressive properties depending on cellular context and experimental conditions. SIRT2 exists in the cytoplasm, deacetylates tubulin, and was observed to have tumor-promoting activity in several studies. SIRT6 localizes in the nucleus and deacetylates histone H3K9 and H3K56 to maintain genome stability and telomere function. This study points to the potential of studying sirtuins in relation to breast cancer. Above all SIRT2 appears to play a pro-tumorigenic role, which could serve as a biomarker of poor prognosis. 